\title{
Is Visual Motion Processing Vulnerable to Early Aging?
}

\author{
Ryan McBain ${ }^{1}$, Dan Norton ${ }^{1}$ and Yue Chen ${ }^{*}, 1,2$ \\ ${ }^{1}$ Mailman Research Center, McLean Hospital, 115 Mill Street, Belmont, MA 02478, USA \\ ${ }^{2}$ Department of Psychiatry, Harvard Medical School, 25 Shattuck Street, Boston, MA 02115, USA
}

\begin{abstract}
While many behavioral capacities decline with age, few can be readily noticed in the early stages of aging. Visual motion processing is particularly sensitive to aging; yet it is unclear whether decline in motion processing can be used to index the visual aging process in its early stages. We examined performance in 63 healthy individuals on a series of motion-related visual tasks, in a large age range (21-80 years-old). Three psychophysical tasks were used: dynamic contrast detection (a functional precursor to motion processing), contrast-based velocity discrimination (motion processing dependent upon contrast signal), and velocity discrimination (motion processing independent of contrast modulation). Additionally, cognitive ability was assessed using the WAIS-R (verbal components). Results indicated that visual motion performance began to significantly decline between the 51-60 and 61-70 age groups, and this decline was most pronounced on velocity discrimination tasks. Cognitive performance did not differ across age groups and was not correlated with performance on visual measures. This pattern of results suggests that visual motion processing may be a potential indicator of early aging within the visual system.
\end{abstract}

Keywords: Vision, contrast detection, velocity discrimination, psychophysics, perception.

\section{INTRODUCTION}

Declines in visual functioning across the adult life span are well-documented in the literature (for a review, see [1]). While certain aspects of visual decline implicate ophthalmic deterioration (e.g. decreased density of retinal ganglion cells [2]), an increasing body of evidence points to the existence of a more general decline within visual cortical systems. For example, visually evoked potentials have extended latency and decreased amplitude in the elderly [3-5], and this is thought to be associated with synaptic loss within the striate cortex [6]. Primate studies have further found decreased stimulus selectivity as well as increased spontaneous activity of visual cortical cells $[7,8]$. Performance on sophisticated visual perception tasks, such as motion discrimination, is mediated in both the striate and extrastriate cortices (e.g., [9, $10])$.

While there have been numerous studies on age-related visual decline, relatively fewer have investigated the effects of aging on dynamic visual processing. Recently, there have been deliberate efforts to identify specific age-related changes in sensitivity to visual motion. For example, Billino and colleagues (2008) [11] observed increased thresholds (declined performance) for translational and biological motion perception, but not radial motion perception, across life span. This suggests that specialized processing mechanisms may differ in their vulnerability to physiological changes of aging. Along similar lines, a study by Snowden and Kavanagh (2006) [12] found that elderly individuals are particularly impaired on slow (as compared to fast) speeds of motion detection and coherent motion tasks. This was in

*Address correspondence to this author at the Room G06, Centre Bldg., 115 Mill Street, Belmont, MA 02478, USA; Tel: 617-855-3615; E-mail: ychen@mclean.harvard.edu contrast to their finding that elderly adults were impaired on a speed discrimination task at both slow and fast speeds of motion. Declined performance on motion detection has also been documented in a more recent study [13].

Each of these investigations has provided important insights regarding decline in dynamic vision. However, it remains to be seen whether certain aspects of visual motion processing may be vulnerable to "early aging". Many perceptual studies have compared only young adults with elderly adults (e.g. [12]), leaving room for the possibility that performance decline may occur during an "early aging" stage, such as during middle-age. Most behavioral capacities do not undergo noticeable changes in the early stages of aging, making them difficult to be detected and assessed. In this regard, visual motion processing may be particularly useful in that this capacity experiences a greater degree of change throughout adult life, and could potentially be utilized for studying early aging processes [14]. Moreover, many aspects of motion processing such as velocity discrimination and dynamic contrast detection have functional implications in various aspects of daily life [15, $16]$.

In the present study, we compared individuals across the adult life span (21-80) on dynamic contrast detection and velocity discrimination, two visual tasks on which elderly adults exhibit degraded performance [17, 18]. In order to inspect the relationship between these two domains of processing, we also included an additional task in which velocity discrimination was modulated by visual contrast. While speed perception may be mediated by one or more motion mechanisms [19-21], tests in this study were done only using speeds of motion 10 degrees $/ \mathrm{sec}(5 \mathrm{~Hz}, 0.5$ cycles/degree) and greater, as such targeting the visual mechanisms which process faster motion speeds. Based upon previous findings $[17,22]$, we hypothesized that the elderly 
group in this study would be relatively impaired on all tasks. Furthermore, with reference to a previous finding of ours that individuals in their fifties already begin to exhibit deficient perception of relative velocity [14], we hypothesized that the middle-aged groups may be differentially impaired on the velocity discrimination task.

\section{MATERIALS AND METHODOLOGY}

\section{Participants}

Sixty-three individuals, ranging from 21-80 years-old, participated in the study. The age range was divided into six decadal age groups - 21-30, 31-40, 41-50, 51-60, 61-70, and 71-80. Subjects were evenly distributed in these groups, with approximately ten in each. Groups were similar in terms of verbal IQ, as measured by the WAIS-R [23], as well as years of education. Participants were local denizens of the Greater Boston area, recruited through advertisements. Inclusion criteria were defined as: (1) age between 21-80 years-old, (2) verbal IQ greater than 70, (3) visual acuity of at least 20/30, as measured by the Rosenbaum Pocket Screener, (4) no disorders in ocular health such as astigmatism or glaucoma (5) no recent history of drug or alcohol abuse (i.e., within the past six months), (6) no history of organic brain disease or psychiatric illness. Prospective participants were screened using the Structured Clinical Interview - Non-Patient Version [24]. The protocol was approved by the Institutional Review Board of McLean Hospital, and written informed consent was attained from each participant.

\section{Procedures}

Participants were tested over one two-hour session, during which all visual tasks and cognitive measures were administered. Visual tasks were run using VisionShell software on a Macintosh Quadra 650. Visual stimuli were displayed on an Apple High-Resolution Monochrome Monitor adapted with a luminance attenuator, which permitted fine contrast gradations. The stimulus for all tasks was a vertical sinusoidal contrast grating subtending 13 degrees of visual angle, with a mean luminance of $35 \mathrm{~cd} / \mathrm{m}^{2}$. Participants were seated in a darkened room at a distance of $61 \mathrm{~cm}$ from the monitor and performed visual tasks binocularly.

There were three visual perception tasks - (1) dynamic contrast detection [25, 26], (2), contrast-based velocity discrimination [27, 28], and (3) velocity discrimination independent of contrast modulation [29, 30]. Detailed instructions and practice trials were administered before formal data collection. Each task utilized a two-alternative forced choice staircase method: Upon three consecutive correct responses by participants, level of stimulus strength (independent variable) increased, and with each incorrect response, level of stimulus strength decreased. Performance on each task was measured by subjects' perceptual thresholds, defined as the minimum stimulus strength level required for participants to achieve $79 \%$ accuracy. To facilitate comparisons of visual and cognitive performance, thresholds were converted to sensitivity indices (log of $1 /$ threshold) for all analyses, unless otherwise specified. Thus, higher scores (i.e., lower thresholds) represent better performance. For all task conditions, a fixation cross was provided at the center of the visual field. Within a given trial, stimuli were presented for $300 \mathrm{msec}$, and separated by a 500 msec inter-stimulus interval.

\section{Dynamic Contrast Detection}

Subjects indicated which of two successive time intervals (first or second) contained a vertical sinusoidal grating. Frequency of the grating was set as 0.5 cycles/degree, and temporal modulation was set to $5 \mathrm{~Hz}$, which resulted in target movement of 10 degrees/second, either to the right or to the left. The threshold was defined as the minimum contrast level required for subjects to reach $79 \%$ accuracy.

\section{Contrast-based Velocity Discrimination}

Subjects determined which of two successively presented vertical sinusoidal gratings moved faster. The velocity difference between the gratings was fixed at $40 \%$ (7 Hz. v. 5 $\mathrm{Hz}$ ). As in contrast detection, thresholds were defined as minimum contrast level at which participants reached $79 \%$ accuracy.

\section{Velocity Discrimination}

Subjects indicated which of two successively presented vertical sinusoidal gratings moved faster. For the initial trial, velocity difference between gratings was set to $100 \%(10 \mathrm{~Hz}$ v. $5 \mathrm{~Hz}$ ). The velocity difference changed based upon subjects' responses. Spatial frequency (0.5 cycles/degree) and contrast $(10 \%)$ were fixed. Thresholds were defined as minimum velocity difference between gratings at which subjects achieved 79\% accuracy.

\section{RESULTS}

One-way ANOVAs with age as the independent variable (21-30, 31-40, 41-50, 51-60, 61-70, 71-80) showed significant effects for this factor in all three tasks (dynamic contrast detection, $\mathrm{F}=8.395, \mathrm{p}<0.001 ;$ contrast-based velocity discrimination, $\mathrm{F}=9.593, \mathrm{p}<0.001$; velocity discrimination, $\mathrm{F}=8.931, \mathrm{p}<0.001)$. Independent of age brackets, regression analyses showed significant correlations between age and performance, which ranged from $r=-0.37$ to $r=-0.45$ and were significant for all visual tasks $(\mathrm{p}<0.01)$, and as such indicates that declined performance with age (see Fig. 1).

To inspect visual decline across age range more closely, performance was also compared in abutting age groups. On this measure, performances differed significantly $(\mathrm{p}<0.05)$ for all motion perception tasks between the age ranges of 5160 and 61-70. For the contrast-based velocity discrimination, there was also a significant group difference $(p=0.032)$ when comparing age group 31-40 to age group 41-50. With Bonferroni corrections, comparisons between age groups 5160 and 61-70 remained significant for two tasks - velocity discrimination $(p=0.002)$ and contrast based velocity discrimination $(p=0.027)$, indicating more pronounced performance declines in these task conditions.

Using Cohen's $d$, effect sizes (ES) were calculated in order to compare visual performance in each age group (e.g. 41-50 years old) relative to the preceding age group (e.g. 3140 years old) (see Table 1). Consistent with the aforementioned analyses, ES was large (above 0.90) when 

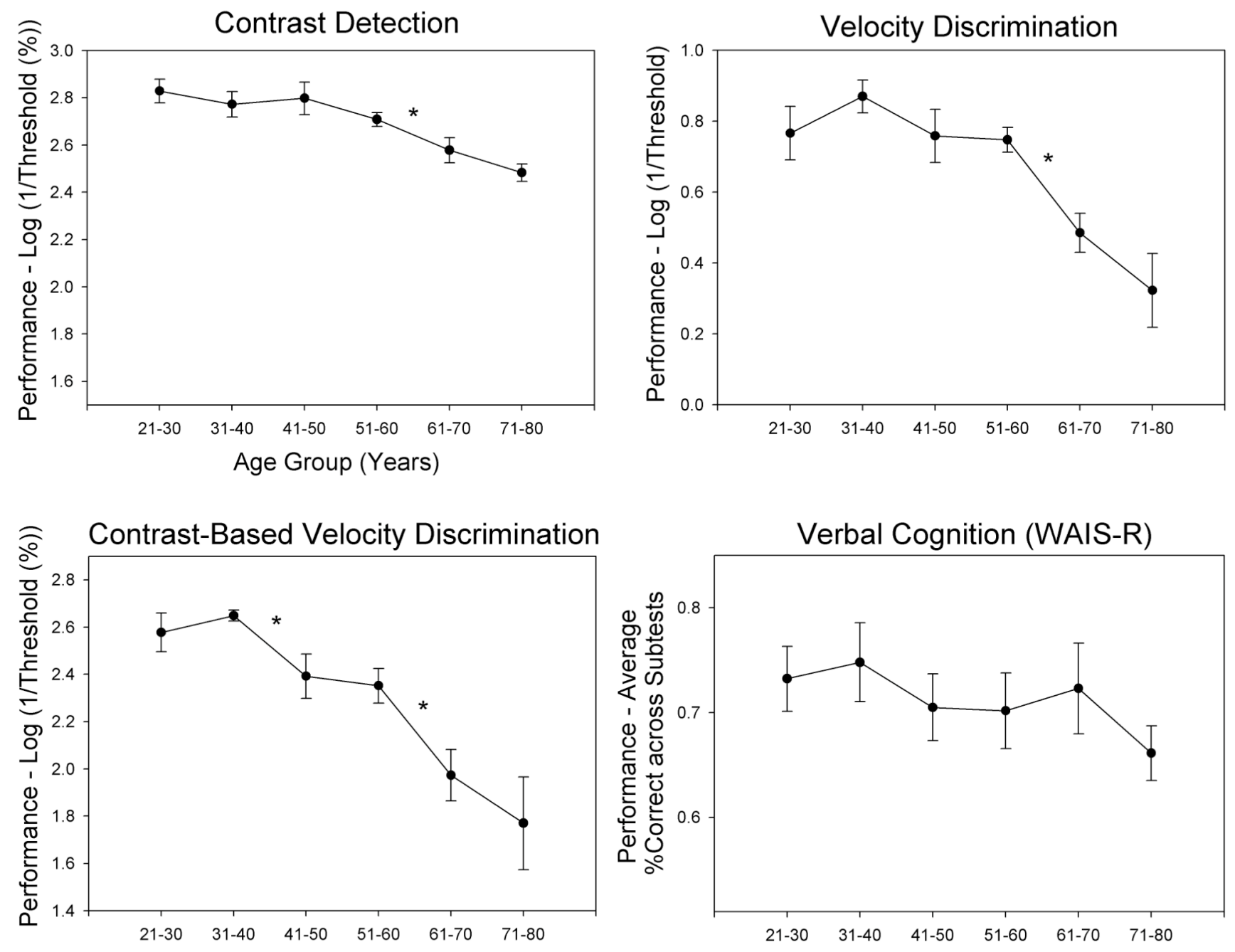

Fig. (1). Performance across age groups on each visual task, as well as on the comparison cognitive measure (WAIS-R). The x-axis indicates age group; the $\mathrm{y}$-axis indicates the averaged sensitivity or accuracy of each age group for the task/condition. For the visual tasks, sensitivity was defined as $\log$ of the reciprocal of the threshold. The unit for the threshold of contrast detection and contrast based velocity discrimination tasks was $\%$ of contrast. The threshold of the velocity discrimination task was \% of velocity difference, or unitless. Error bars indicate \pm 1 standard error. Asterisks $(*)$ indicate significant differences between abutting age groups $(\mathrm{p}<0.01)$.

comparing persons aged 61-70 with those aged 51-60, and largest for the contrast based velocity discrimination (ES = $1.34)$ and velocity discrimination $(\mathrm{ES}=1.87)$ tasks.

\section{Cognitive Measures}

One-way ANOVAs with age showed that neither absolute (raw) nor scaled (relative) scores on verbal components of the WAIS-R differed significantly across the six age groups $(\mathrm{p}>0.05)$. Furthermore, correlations between scores on visual and cognitive measures were all nonsignificant $(\mathrm{p}>0.05)$. Additionally, age-related decline on visual motion tasks remained highly significant when cognitive measures were used as covariates $(p<0.001)$.

\section{DISCUSSION}

While a few studies have found that elderly adults are unimpaired or may even improve on specific types of motion processing (e.g., [31]), this study shows a pervasive decline on dynamic contrast detection and velocity discrimination tasks. Moreover, the data also indicate that the onset and magnitudes of these declines differed among tasks. Dynamic contrast detection gradually declined beginning in the 51-60 year-old age group. In comparison, there was an unexpected performance decline on velocity discrimination tasks between the 31-40 and the 41-50 age groups.

A more acute performance decline on velocity discrimination tasks was also present in the 61-70 age group: Comparing the 51-60 and 61-70 age groups, effect sizes on these two tasks were 1.34 and 1.87 , as compared with 0.93 for dynamic contrast detection. These sharper declines were further evidenced by additional statistical comparisons (see Results), which found significant differences between age groups on the velocity discrimination tasks, but not on dynamic contrast detection, when more stringent criteria (i.e., those based on the Bonferroni correction procedure) 
Table 1. Effect Sizes between Abutting Age Groups

\begin{tabular}{|c|c|c|c|c|}
\hline & Dynamic Contrast Detection & Contrast-Based VD & Velocity Discrimination (VD) & Verbal Cognition (WAIS-R) \\
\hline \hline $21-30$ vs. $31-40$ & 0.35 & -0.40 & -0.58 & -0.22 \\
\hline $31-40 v s .41-50$ & -0.15 & $1.42^{*}$ & 0.69 & 0.65 \\
\hline $41-50$ vs. $51-60$ & 0.55 & 0.17 & 0.07 & 0.04 \\
\hline $51-60 v s .61-70$ & $0.93 *$ & $1.34^{*}$ & $1.87^{*}$ & -0.23 \\
\hline $61-70 v s .71-80$ & 0.64 & 0.47 & 0.72 & 0.76 \\
\hline
\end{tabular}

*indicate significant differences between abutting age groups $(\mathrm{p}<0.05)$.

were imposed. Differential aging on dynamic visual processing tasks has been shown previously [11,32, 33]. Our data contribute to this literature by showing different onsets and magnitudes of age-related declines on dynamic contrast detection and velocity discrimination.

Contrast- and motion-based visual processing primarily occurs within the striate and extrastriate cortices. Comparing old to young adults, neuroimaging studies have found decreased responses to visual stimuli in these brain areas [34-36]. These decreased responses may result in inadequate visual inputs for perceptual and cognitive tasks. Our data, besides offering behavioral evidence consistent with this interpretation, suggest that age-related changes in the perception of motion are not closely associated with the verbal cognitive ability across life span.

Overall, our results indicate that visual processing of dynamic information declines relatively early across life span. On all motion perception measures, persons in their $6^{\text {th }}$ decade of life exhibited degraded performance relative to younger age groups. A recent report by Snowden and Kavanagh (2006) [12] similarly found age-related declined performance on visual motion tasks prior to persons' 70 s. The study by Snowden and Kavanagh (2006) demonstrated significant performance differences between the two disparate age groups - individuals 17-30 and 54-71 yearsold; yet, it was unclear from this result precisely when the performance decline occurred. The present study examined how motion perception is incrementally affected across the entire adult life span.

Declined performance on velocity discrimination in middle-aged individuals has two primary implications. First, it suggests that visual response to motion signals is vulnerable to, and thus can potentially be used as a tool for assessing, early aging. Second, many daily activities, especially those of dynamic nature such as driving, rely on judgments of the relative velocity of moving targets. Declined motion discrimination in this early aging stage, particularly on the contrast-based task, suggests that the perceptual capacities pertaining to dynamic vision may begin to change earlier in life than previously thought. This functional decline indicates that additional visual signal strength may be needed in order to engage in certain daily activities like driving. When providing visual environments optimized for different age groups, a declined capacity to perceive moving targets should be taken into consideration prior to what is traditionally classified as 'elderly adulthood'.

\section{CONCLUDING REMARKS}

The current study contains a couple limitations, which should be mentioned. First, our sample size, when separated into decadal groups, was relatively small. Thus, the results, while generally producing robust effect sizes, should be confirmed in a larger sample. Secondly, the comparisons between visual tasks and verbal cognitive measures were useful; however, introducing a different set of cognitive measures - i.e., those which directly depend on visual inputs - would have provided additional insight into the relationship between visual and cognitive declines.

In summary, the results of this study suggest that visual motion processing declines relatively early across life span, generally beginning in persons' fifties to sixties, and may be an early marker of a more global decline in the visual system.

\section{ACKNOWLEDGEMENTS}

This study was supported in part by a grant from the NIH, through the Harvard School of Public Health.

\section{REFERENCES}

[1] Haegerstrom-Portnoy G, Schneck ME, Brabyn JA. Seeing into old age: vision function beyond acuity. Optom Vis Sci 1999; 76(3): 141-58.

[2] Harman A, Abrahams B, Moore S, Hoskins R. Neuronal density in the human retinal ganglion cell layer from 16-77 years. Anat Rec 2000; 260(2): 124-31.

[3] Li RW, Edwards MH, Brown B. Variation in vernier evoked cortical potential with age. Invest Ophthalmol Vis Sci 2001; 42(5): 1119-24.

[4] Lorenzo-Lopez L, Amenedo E, Pascual-Marqui RD, Cadaveira F. Neural correlates of age-related visual search decline: A combined ERP and sLORETA study. Neuroimage 2008; 41(2): 511-24.

[5] Tobimatsu S. Aging and pattern visual evoked potentials. Optom Vis Sci 1995; 72(3): 192-7.

[6] Fiorentini A, Porciatti V, Morrone MC, Burr DC. Visual ageing: unspecific decline of the responses to luminance and colour. Vision Res 1996; 36(21): 3557-66.

[7] Schmolesky MT, Wang Y, Pu M, Leventhal AG. Degradation of stimulus selectivity of visual cortical cells in senescent rhesus monkeys. Nat Neurosci 2000; (4): 384-90.

[8] Yu S, Wang Y, Li X, Zhou Y, Leventhal AG. Functional degradation of extrastriate visual cortex in senescent rhesus monkeys. Neuroscience 2006; 140(3): 1023-9.

[9] Maunsell JHR, Newsome WT. Visual processing in monkey extrastriate cortex. Annu Rev Neurosci 1987; 10: 363-401.

[10] Culham J, He S, Dukelow S, Verstraten FA. Visual motion and the human brain: what has neuroimaging told us? Acta Psychol (Amst) 2001; 107(1-3): 69-94. 
[11] Billino J, Bremmer F, Gegenfurtner KR. Differential aging of motion processing mechanisms: Evidence against general perceptual decline. Vision Res 2008; 48(10): 1254-61.

[12] Snowden RJ, Kavanagh E. Motion perception in the ageing visual system: minimum motion, motion coherence, and speed discrimination thresholds. Perception 2006; 35(1): 9-24.

[13] Bennett PJ, Sekuler R, Sekuler AB. The effects of aging on motion detection and direction identification. Vision Res 2007; 47(6): 799809.

[14] Bidwell LC, Holzman PS, Chen Y. Aging and visual motion discrimination in normal adults and schizophrenia patients. Psychiatry Res 2006; 145(1): 1-8.

[15] Scialfa CT, Guzy LT, Leibowitz HW, Garvey PM, Tyrrell RA. Age differences in estimating vehicle velocity. Psychol Aging 1991; 6(1): 60-6.

[16] Wood JM, Owens DA. Standard measures of visual acuity do not predict drivers' recognition performance under day or night conditions. Optom Vis Sci 2005; 82(8): 698-705.

[17] Owsley C, Sekuler R, Siemsen D. Contrast sensitivity throughout adulthood. Vision Res 1983; 23(7): 689-99.

[18] Scialfa CT, Kline DW, Lyman BJ, Kosnick W. Age differences in judgments of vehicle velocity and distance. Proceedings of the Annual Meeting of the Human Factors Society. New York: Human Factors Society 1987; 558-61.

[19] Cavanagh P, Mather G. Motion: the long and short of it. Spat Vis 1989; 4(2-3): 103-29.

[20] van Boxtel JJ, van Ee R, Erkelens CJ. A single system explains human speed perception. J Cogn Neurosci 2006; 18(11): 1808-19.

[21] Balas B, Sinha P. A speed-dependent inversion effect in dynamic object matching. J Vis 2009; 9(2): 161-3.

[22] Raghuram A, Lakshminarayanan V, Khanna R. Psychophysical estimation of speed discrimination. II. Aging effects. J Opt Soc Am A Opt Image Sci Vis 2005; 22(10): 2269-80.

[23] Wechsler D. Manual for the Adult Intelligence Scale-Revised. New York: Psychological Corporation 1981.
[24] First MB, Spitzer RL, Gibbon M, William JB. Structure Clinical Interview for DSM -IV-TR Axis I Disorders - Non-patient Edition (SCID-I/NP, 11/2002 revision) New York, NY Biometric Research Department, New York State Psychiatric Institute 2002.

[25] Robson JG. Spatial and temporal contrast sensitivity functions of the visual system. J Opt Soc Am 1966; 56: 1141-2.

[26] Kelly DH. Motion and vision. II. Stabilized spatio-temporal threshold surface. J Opt Soc Am 1979; 69(10): 1340-9.

[27] Pasternak T. Discrimination of differences in velocity and flicker rate depends on directionally selective mechanisms. Vision Res 1987; 30: 625-60.

[28] Chen Y, Palafox GP, Nakayama K, Levy DL, Matthysse S, Holzman PS. Motion perception in schizophrenia. Arch Gen Psychiatry 1999; 56(2): 149-54.

[29] McKee S, Silverman G, Nakayama K. Precise velocity discrimination despite random variations in temporal frequency and contrast. Vision Res 1986; 26(4): 609-19.

[30] Chen Y, Bedell HE, Frishman LJ. The precision of velocity discrimination across spatial frequency. Perceptual Psychophysics 1998; 60(8): 1329-36.

[31] Betts LR, Taylor CP, Sekuler AB, Bennett PJ. Aging reduces center-surround antagonism in visual motion processing. Neuron 2005; 45(3): 361-6.

[32] Andersen GJ, Ni R. Aging and visual processing: declines in spatial not temporal integration. Vision Res 2008; 48(1): 109-18.

[33] Habak C, Faubert J. Larger effect of aging on the perception of higher-order stimuli. Vision Res 2000; 40(8): 943-50.

[34] Justino L, Kergoat H, Kergoat MJ. Changes in the retinocortical evoked potentials in subjects 75 years of age and older. Clin Neurophysiol 2001; 112(7): 1343-8.

[35] Levine BK, Beason-Held LL, Purpura KP, et al. Age-related differences in visual perception: a PET study. Neurobiol Aging 2000; 21(4): 577-84

[36] Peiffer AM, Hugenschmidt CE, Maldjian JA, Casanova R, Srikanth $\mathrm{R}$, Hayasaka S, et al. Aging and the interaction of sensory cortical function and structure. Hum Brain Mapp 2007; 30(1): 228-40.

(C) McBain et al.; Licensee Bentham Open.

This is an open access article licensed under the terms of the Creative Commons Attribution Non-Commercial License (http://creativecommons.org/licenses/by$\mathrm{nc} / 3.0 /$ ), which permits unrestricted, non-commercial use, distribution and reproduction in any medium, provided the work is properly cited. 